\title{
A refractive index-matched facility for fluid-structure interaction studies of pulsatile and oscillating flow in elastic vessels of adjustable compliance
}

\author{
S. Burgmann $\cdot$ S. Große $\cdot$ W. Schröder $\cdot$ \\ J. Roggenkamp · S. Jansen · F. Gräf · \\ M. Büsen
}

Received: 17 October 2008/Revised: 24 April 2009/Accepted: 5 May 2009/Published online: 23 May 2009

(C) The Author(s) 2009. This article is published with open access at Springerlink.com

\begin{abstract}
The flow field in the respiratory and vascular system is known to be influenced by the flexibility of the walls. However, up to now, most of the experimental biofluidic investigations have been performed in rigid models due to the complexity and necessity of optical access. In this paper, a facility and measurement techniques for studying oscillating and pulsatile flow in elastic vessels will be described. The investigated vessel models have been adapted such that fluid-mechanical and structure-mechanical characteristics represent realistic blood flows in medium blood vessels. That is, characteristic parameters, i.e., the Reynolds and Womersley number, as well as mechanical properties of the flexible wall, i.e., the Young's modulus and the material compliance, have been chosen to reasonably represent realistic flow conditions. First, a method to manufacture elastic models, which mimic the structure-mechanical properties of vascular vessels is described. The models possess a tunable compliance and are made of transparent polydimethylsiloxane. Second, the experimental setup of the flow facility will be elucidated. The flow facility allows to mimic pulsatile flow at physiologically relevant Reynolds and
\end{abstract}

S. Burgmann and S. Große contributed equally to this work.

S. Große (ه)

Laboratory for Aero and Hydrodynamics,

Delft University of Technology, 2628 CA Delft,

The Netherlands

e-mail: sebastian.grosse@rwth-aachen.de

S. Burgmann · W. Schröder · J. Roggenkamp - S. Jansen ·

F. Gräf · M. Büsen

Institute of Aerodynamics, RWTH Aachen University,

52062 Aachen, Germany

e-mail: s.burgmann@aia.rwth-aachen.de
Womersley numbers. The precise form of the flow cycle can individually be controlled. Water/glycerine is used as flow medium for refractive index matching particle image velocimetry (PIV) measurements. The PIV recordings not only allow to assess the mean cross-sectional flow field but also further enable to simultaneously detect the movement of the flexible wall. Additionally, the local wall-shear stress can be obtained from the single-pixel line resolved near-wall flow field. To confirm the flow conditions of the oscillatory laminar flow inside the flow facility and to evaluate the ability to assess the flow field, measurements in a straight, uniform diameter, rigid Plexiglas pipe under identical conditions to those of the oscillating flow in the flexible vessel have been performed. The measurements of oscillating flow in the rigid pipe corroborate the experimentally obtained flow field and the wall-shear stress to well confirm Womersley's analytical solution and thereby evidence the quality of the flow facility and of the measurement techniques. To further study the detectability of the vessel deformation, oscillating flow at Reynolds numbers based on the non-dilated vessel diameter $D$ and peak velocities $R e_{D}$ ranging from 1,000 to 1,750 and at Womersley numbers $\alpha$ ranging from 5 to 17.5 has been investigated in an elastic vessel.

\section{Introduction}

In most of the biomechanical flows, e.g., respiratory and vascular flows, the flexibility of the walls is expected to possess significant effects on the evolving flow field. It has been shown that flexibility in general and especially illness-related locally reduced flexibility strongly effects the wall friction and intensifies the stress on local pathological structures. While recently performed numerical biofluidic 
investigations accounted for the flexibility of the vessels, experimental investigations of pulsatile flows in elastic vessels are far from being complete although quite some effort has been made for more than 50 years. However, there exists a great demand for an experimental database available for a validation of the numerical results of fluidstructure interacting flow fields.

An analytical solution for the velocity distribution due to an oscillating flow in rigid and slightly elastic pipes has been introduced in Womersley (1957a, b), respectively. This simple theory by Womersley has been widely used in the past to calculate the velocity distribution based on pressure measurements, e.g., in Hale et al. (1955) and Mijovic and Liepsch (2003). Also to cover larger deformations of the elastic vessel occurring e.g., in human arteries, Womersley's theory has been extended by McDonald (1955). In spite of these extensive investigations the coherence of the pressure development and the vessel deformation in real elastic vessels is still not comprehensively clarified.

Recently, optical measurement techniques have been introduced to directly measure the velocity distribution in elastic vessels instead of calculating the velocity based on pressure measurements, like in Womersley (1955). Using phase-locked laser Doppler velocimetry (LDV) the elasticity has been shown to play a major roll concerning the flow field (Mijovic and Liepsch 2003). Furthermore, the applicability of particle image velocimetry (PIV) to capture instantaneous velocity fields in elastic vessels has been discussed in Eguchi et al. (2003). Nevertheless, a detailed description of the fluid-structure interaction in elastic vessels is still lacking, especially as a reliable database to validate CFD results. As a first step towards a comprehensive analysis of physiological flows and of the fluidstructure interaction the oscillating flow field in an elastic circular vessel is to be investigated.

In this paper, a flow facility and data evaluation techniques for the detailed study of pulsatile and oscillating flow in transparent elastic vessels will be intensively described. The flow facility allows to mimic pulsatile and oscillating flow at physiologically relevant Reynolds and Womersley numbers and the exact form of the flow cycle can individually be controlled.

An elastic tube made of transparent poly-silicone is manufactured taking into account fluid-mechanical and physiological parameters. That is, the vessel models have been adapted such that their characteristics represent realistic flow in medium blood vessels. The structuremechanical and geometrical properties of this tube are validated by several measurement techniques.

In conjunction with the refractive index (RI) adapted flow facility the vessels become optically transparent and allow to study the evolving flow field by means of PIV. The technique has already been successfully applied to perform measurements of the flow field evolving in a realistic, transparent-but rigid - silicone model of the upper human airways (Große et al. 2007, 2008).

The tube is inserted into a piston-pump-driven test section, which can generate an oscillating or pulsatile flow field. The pumping frequency and the piston stroke can be adjusted to achieve the desired Womersley and Reynolds number. The applied techniques allow to detect the flow field inside the tube and the dilatation of the tube simultaneously. Furthermore, it is possible to derive the wallshear stress distribution from the very near-wall velocity gradient.

The structure of the paper is as follows. First, a brief overview of the mechanical background of oscillating laminar pipe flow will be given to define some of the relevant fluid-mechanical and structure-mechanical characteristics. In Sect. 3, the fabrication and validation of the elastic arterial models with tunable compliance made of transparent poly-silicone (PDMS) are discussed in detail.

Subsequently, the experimental facility, in which the model is used, will be described in Sect. 4. In Sect. 5, the data evaluation, i.e., the determination of the velocity field using PIV, the determination of the vessel dilatation by image pattern detection, and the assessment of the wallshear stress via near-wall velocity gradient by means of single pixel-line correlations will be discussed.

Finally, in Sect. 6 results from validation measurements at oscillating flow will be discussed. A short conclusion in Sect. 7 wraps up the paper.

\section{Mechanical background}

To adequately model biomedical flows, e.g., the pulsatile flow in arteries, not only fluid mechanical but also physiological parameters have to be satisfied in the experiment. These physiological parameters include, for instance, the vessel compliance $C$, the Reynolds number $R e_{D}$, the Womersley number $\alpha$, and the ratio of the fluid velocity to the wave propagation speed $u / c$. The Womersley number and the Reynolds number define the mass flow in the vessel and therefore the pressure gradient which governs the vessel compliance $C$. The Moens-Korteweg formula describes the wave propagation velocity

$c=\sqrt{\frac{E h}{2 \rho\left(R_{a}-h\right)}}$

as a function of Young's modulus $E$, the outer radius $R_{a}$, and the wall thickness of the vessel $h$, and the density of the fluid $\rho$. On the one hand, the compliance is directly related to the wave propagation velocity and, on the other hand, it 
is defined as the rate change of the vessel cross-section $A$ with respect to the pressure $p$ normalized by the nondilated vessel cross-section $A_{0}$.

$C=\frac{1}{\rho c^{2}}=\frac{\partial A / \partial p}{A_{0}} \approx \frac{2 \partial\left(R_{a}-h\right) / \partial p}{\left(R_{a}-h\right)}, \quad[C]=1 / P a$.

The vessel compliance can be tuned by varying Young's modulus $E$, the outer radius of the vessel $R_{a}$, and the wall thickness $h$. In the current experiment, which is designed to model pulsatile blood flow in arteries, an outer radius of the vessel of $R_{a}=12.0 \mathrm{~mm}$ has been chosen in accordance with values in human arteries. The corresponding Reynolds number

$R e_{D}=\frac{2 u\left(R_{a}-h\right) \rho}{\eta}$

is $R e_{D}=1,750$ with $u$ being the fluid velocity and $\eta$ the dynamic viscosity of the fluid. Using the Moens-Korteweg formula and a ratio of the fluid velocity to the wavepropagation speed $u / c=0.043$ the wall thickness $h$ can be determined. To adapt the model characteristics to the physiological boundary conditions the required wall thickness of the elastic tube was calculated to be $h=1.59 \mathrm{~mm}$.

\section{The elastic vessel}

\subsection{Vessel material and manufacturing procedure}

In the following, some aspects of the material and the manufacturing procedure are considered. Details about the probes and how the mechanical properties are determined will be given in the next section.

\subsubsection{Material}

The elastic tubes are manufactured from GE two-component silicon rubber RTV615 (A+B). This material has been chosen due to its excellent mechanical, chemical, and optical properties (Lötters et al. 1996, 1997a, b; van Hout et al. 2003). Due to the low viscosity of the uncured material it perfectly distributes in the mold. The mechanical properties can be varied in a wide range by an adapted manufacturing procedure. Mechanical tests performed in this study showed the mechanical hysteresis between loading and unloading cycles to be negligible.

A shrinkage after an isothermal poly-addition during curing of the material is in the order of $0.2 \%$ (Dow Corning Corporation 1991). That is, the geometrical properties such as diameter and wall thickness can be precisely adjusted without accounting for shrinkage. RTV615 is characterized by good chemical resistance against many fluids such as water and glycerine, i.e., the fluids used in these measurements.

The optical transparency of the material and its RI of 1.406 are ideal for optical measurement techniques such as PIV. However, the RI of the fluid needs to be adjusted. Several tests showed a water/glycerine mixture with 60.7 weight percent glycerine to fulfill these requirements. A further discussion is given in Sect. 4.2.

\subsubsection{Manufacturing procedure}

A centrifugal device is used to manufacture the elastic tubes, an image of which is given in Fig. 1a. The device consists of a precision steel tube (DIN 2391) with an inner diameter of $24 \mathrm{~mm}$, which can be rotated at up to $f=1,500 \mathrm{rpm}$. The concentricity of the outer steel tube diameter is determined to be within $0.025 \mathrm{~mm}$ over the length of $0.6 \mathrm{~m}$. The nominal tolerance in the thickness of the precision steel tube is less than $\pm 0.08 \mathrm{~mm}$ at the diameter/wall thickness. Furthermore, the horizontal alignment was determined to be within $\pm 0.05^{\circ}$. Note, due to the high-centrifugal acceleration, already slight differences in the radius influence the resulting uniformity of the wall thickness of the cured elastic tube. Furthermore, any disturbance from a horizontal position causes the liquid silicone to tend towards one end of the tube.

To better remove the elastic tube after curing the inner surface of the steel tube is primered and polished using
Fig. 1 a Image of the centrifugal manufacturing device. b Elastomer vessels, pillar for dynamic determination of Young's modulus, tensile specimen
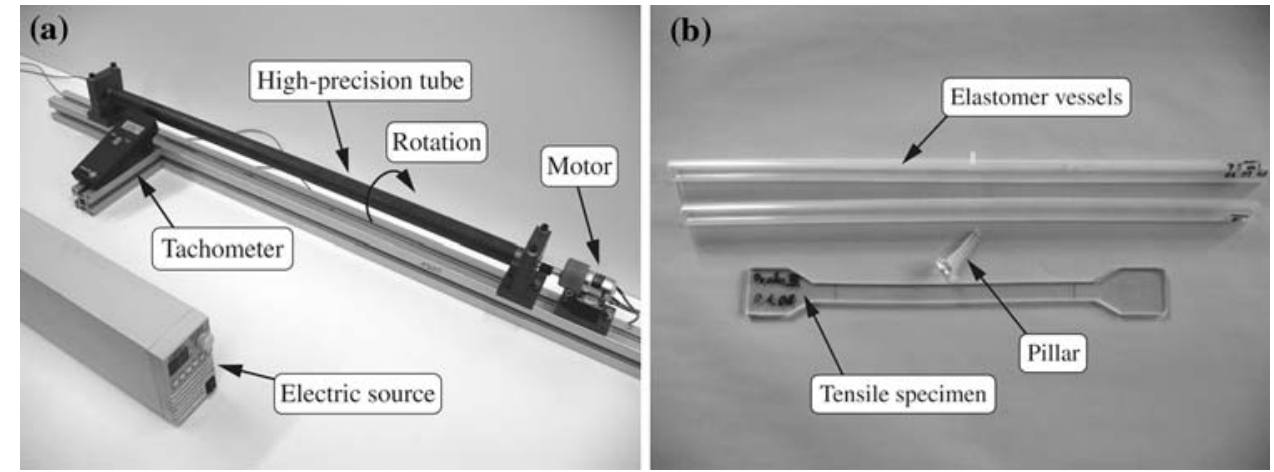
standard wax coating to enhance the surface quality of the steel tube even further.

The two components of the RTV615 silicone are manually mixed at a 10:1 silicone to curing-agent weight ratio. Note that, by varying the silicone to curing-agent ratio from 5:1 to $15: 1$, the Young's modulus can be tuned by approximately a factor of five (Armani and Liu 1998).

To obtain a homogeneous distribution of the material, the RTV615 mixture needs to be accordingly stirred and degasified. At temperatures below $25^{\circ} \mathrm{C}$ the material does not cure and can be easily cast. To achieve a homogeneous distribution of the PDMS along the complete inner surface of the steel tube, first the centrifugal device is operated at a rotation speed of $f=1,500 \mathrm{rpm}$ for approximately $1 \mathrm{~h}$ at an ambient temperature lower than $25^{\circ} \mathrm{C}$. Subsequently, the temperature is increased to $35^{\circ} \mathrm{C}$ and the tube is rotated at $f=1,500 \mathrm{rpm}$ for another $4-5 \mathrm{~h}$. Then, the material achieves a curing state such that a further rotation is no longer necessary, i.e., the material reaches an elastic rubber-like state. After a total curing time of $24 \mathrm{~h}$, the elastic tube can be easily removed using some lubricant such as glycerine.

The temperature during the curing cycle strongly influences the resulting mechanical properties of the material. Curing at $35^{\circ} \mathrm{C}$ for $12 \mathrm{~h}$ results in a Young's modulus of approximately $E=0.8 \times 10^{6} \mathrm{~N} / \mathrm{m}^{2}$, whereas a curing at $60^{\circ} \mathrm{C}$ for $4 \mathrm{~h}$ yields $E=2.0 \times 10^{6} \mathrm{~N} / \mathrm{m}^{2}$, both at a $10: 1$ silicone to curing-agent mixing ratio. Therefore, curing conditions should be kept very controllable.

Since Young's modulus and all the related mechanical properties depend strongly on the curing procedure, a pair of mechanical probes is cast with each elastic tube (Fig. 1b). Note that, depending on the manufacturing process, a final curing state is not achieved even after $24 \mathrm{~h}$. Measurements of Young's modulus show curing to continue for approximately 7 days.

\subsubsection{Determination of wall thickness}

The wall thickness of the elastic vessel defines the mechanical properties of the model. In other words, it is necessary to be able to adjust this parameter to the desired value by the manufacturing process.

The vessel wall thickness after curing depends on the volume of silicone $V$ inserted into the steel tube. Using a syringe the inserted volume can be determined up to within $2.7 \%$. The length of the wetted surface can be determined up to an error of approximately $\pm 0.3 \%$ at the given total length of $600 \mathrm{~mm}$. Furthermore, $R_{a}$ can be determined to within $0.7 \%$. Thus, assuming the silicone to be perfectly distributed, the resulting wall thickness can be determined to within $\pm 0.4 \%$, i.e., at an adequate accuracy.
3.2 Validation of vessel geometry and mechanical properties

It goes without saying that the knowledge of the geometrical and mechanical properties of the manufactured vessel is essential for a reliable investigation of the flow field and the fluid-structure interaction. This is especially important in the context of a reliable validation database. Following Womersley (1957b), the wall thickness $h$, the radius $R_{a}$, its density $\rho_{\text {vessel }}$, Young's modulus $E$, and the shear modulus $G$ are necessary parameters. Note that, $G=E /\left(1-v^{2}\right)$, where $v$ is the Poisson ratio. In the following, techniques that allow to determine the aforementioned parameters will be discussed.

\subsubsection{Validation of wall thickness}

To validate the wall thickness of the vessel, different techniques have been used. A homogeneous distribution of the wall thickness along the vessel axis and in the circumferential direction is very important since any disturbance causes a non-symmetry of the local compliance and hence, a local deformation of the elastomer.

Therefore, thin circular slices cut from different positions along the vessel axis are analyzed optically using a standard CCD camera. Figure 2a shows an exemplary slice of a vessel. Special care is taken to ensure the slice to be perpendicular to the vessel axis such that the extracted cross-section is not a skewed projection of the vessel, since any misalignment would cause the thickness to be artificially increased (Fig. 2b), e.g., an angle $\alpha=0.5^{\circ}$ between the slice and the vessel axis already yields an error in the determined wall thickness of approximately $+1.0 \%$.

For a better processing of the image, the vessel is colored with non-transparent paint. This increases the local intensity gradient and enables the determination of the vessel contour to within $\pm 1 \mathrm{px}$. The image of the vessel radius is in the order of 300 to $400 \mathrm{px}$ and as such the error in measuring the thickness is about $2.8 \%$.

In Fig. 2c, the values of the wall thickness determined manually from a projection of the slices using an overhead projector have been juxtaposed with results obtained by the above-mentioned procedure. These data evidence a qualitative agreement of the two techniques. The results further show the wall thickness to vary only by $4-7 \%$ in the circumferential direction. Measurements at different positions along the vessel's axis yield very similar findings, i.e., the thickness is shown to be constant along the vessel axis.

It is needless to say that the aforementioned technique requires the vessel model to be destroyed to determine the wall thickness. Therefore, the possibility to measure the local vessel thickness non-destructively using magnetic resonance tomography (MRT) is currently investigated. 


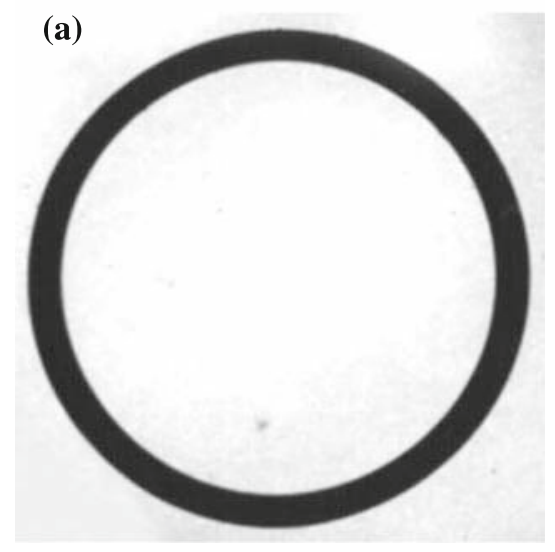

(b)

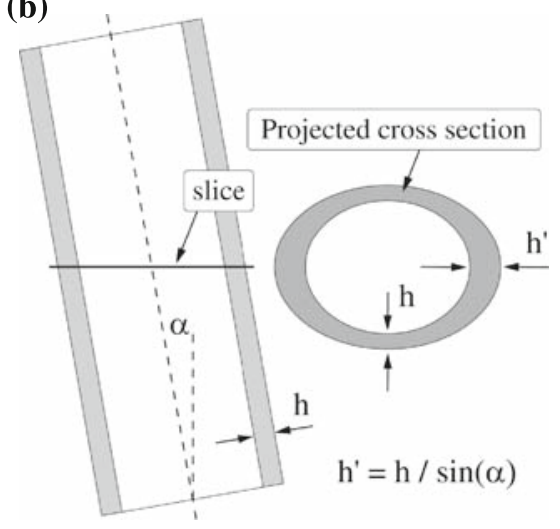

(c)

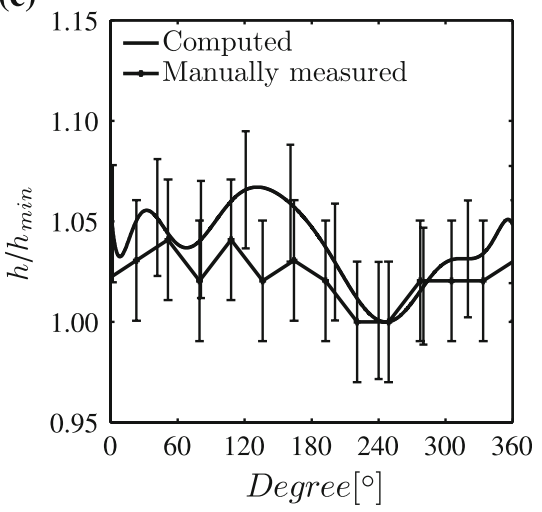

Fig. 2 a Exemplary image of a slice cut out of a vessel. b Illustration of the measurement error when the slice is non-orthogonal to the vessel axis. c Exemplary variation of the local thickness along the circumferential direction measured

\subsubsection{Determination of vessel radius}

The homogeneity of the outer vessel radius $R_{a}$ is determined by the inner tube radius of the centrifugal device. Therefore, the deviation should be within the variance of the precision steel tube. The deviation of the inner diameter of the steel tube is within $\pm 0.08 \mathrm{~mm}$ at the chosen values of outer diameter and thickness of the tube. Measurements of the elastic vessel's diameter after curing using the same technique as described above show the outer diameter to possess the required value of $24 \pm 0.1 \mathrm{~mm}$.

\subsubsection{Determination of material density $\rho_{\text {vessel }}$}

The density of the silicone is determined measuring the weight of a test specimen. The dimensions of the test specimen can be determined to be within 1\%. A highprecision scale PRECISA 160a from PRECISA Gravimetrics $\mathrm{AG}$ with a resolution of $\pm 0.1 \mathrm{mg}$ is used to measure the weight. The probe weight is in the order of 20 $\mathrm{g}$ such that the measurement error is negligible, i.e., the density can be determined within $1 \%$ and is found to be $1,033 \mathrm{~kg} / \mathrm{m}^{3}$.

\subsubsection{Validation of Young's modulus}

The determination of Young's modulus is influenced by the sensitivity of the parameter to the curing cycle. Tests indicated Young's modulus to differ by up to $30 \%$ between probes made of the same charge of silicone but cured at slightly different temperatures. Hence, it is critical to transfer Young's modulus from one specimen to another and we rather suggest to measure the modulus of the actual vessel. At the current state the difference in Young's modulus can be reduced to approximately $5 \%$ by taking special precautions for a constant curing temperature.
Different techniques to measure Young's modulus will be presented in the following. The first test consists of statically extending a tensile specimen. As such, Young's modulus can be calculated from the stress-strain relation. The advantage of this procedure is the possibility to identify the distribution of Young's modulus as a function of stress. Note that, the change in the specimen cross-section needs to be taken into account at high levels of dilatation. A spring balance with a measurement range up to $2.5 \mathrm{~kg}$ is used. The specimen is clamped in a stiff measurement rack. Special care was taken to mount the polymer specimen torsion-free such that only longitudinal stress is applied on the structure. The design of the specimen (Fig. 1b) is such that one-directional stress can be assumed between the measurement positions. The distance of two defined points is measured using a caliper. With the chosen non-dilated length of $L_{0} \approx 200 \mathrm{~mm}$, the dilatation can be determined within $0.2 \%$. Young's modulus is calculated from the linear fit to the measured stress-strain relation. Due to lateral contraction of the material, the non-dilated cross-section $A_{0}$ of the test specimen decreases by approximately $11 \%$, which needs to be accounted for in the calculation of Young's modulus.

Results are given in Fig. 3. Figure 3a, b show the stressstrain relation and the ratio of applying force and strain, respectively, for a tensile specimen, whereas Fig. 3c, d show the distributions for the corresponding elastic vessel model. Both moduli are determined from the linear $(\varepsilon<0.1)$ stress-strain relation and agree within $5 \%$. This is rather an effect of the different temperatures during curing than a problem to accurately determine the parameters. The graphs also show the values for loading and unloading cycles and evidence the material to possess no or a negligible hysteresis.

Note that, Young's modulus is known to vary until PDMS reaches its final curing state. To ensure a constant 
Fig. 3 a Stress-strain relation for the tensile specimen, b Force-strain relation for the tensile specimen, c Stress-strain relation for the 'corresponding' vessel, $\mathbf{d}$ force-strain relation for the 'corresponding' vessel
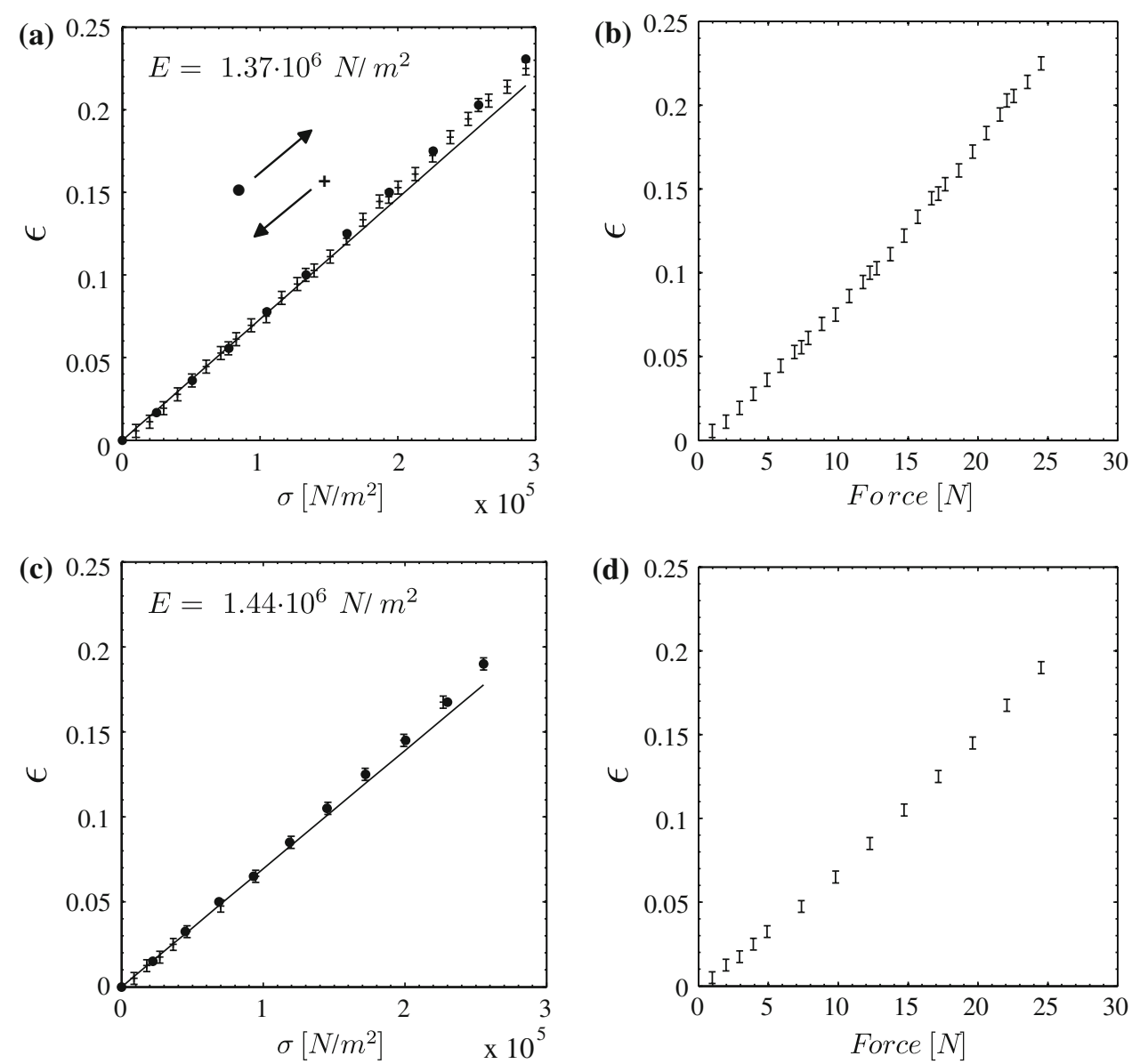

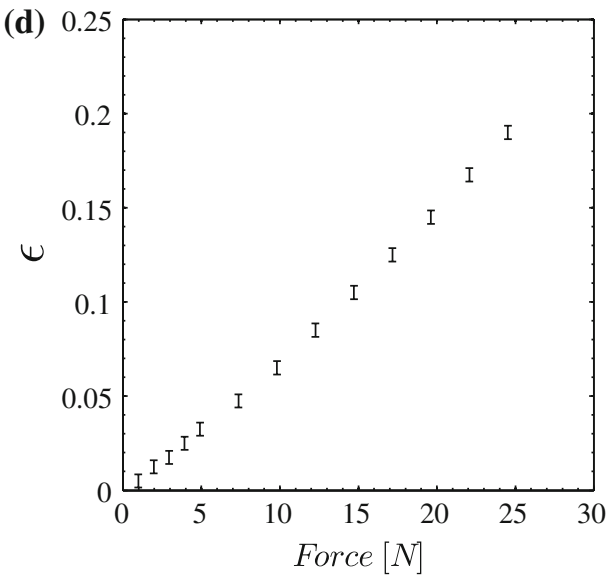

modulus, the vessel is not used for at least 7 days after curing. Measurements of Young's modulus during this time evidences the modulus to reach an asymptotic value. Furthermore, to test the influence of different ambient fluids Young's modulus is evaluated after the probe being immersed in water and water/glycerin mixtures for at least $4 \mathrm{~h}$. The results indicate Young's modulus not to be affected by this treatment.

\subsubsection{Determination of compliance}

To determine the vessel compliance an elastic tube is pressurized using water. The internal pressure is varied by different heights of a water column connected to the vessel. The pressure is measured using a pressure transducer. The dilatation is detected optically by a CCD camera. The outer contour of the vessel can be evaluated using image detection routines. Figure $4 \mathrm{a}$ shows exemplary results for a vessel with an outer diameter $D_{0}=24 \mathrm{~mm}$ and a wall thickness of $h=1.55 \mathrm{~mm}$. The vessel compliance $C=0.091 \% / \mathrm{mmHG}$ is determined from the relationship of the cross-section area and the applied inner pressure. Only the linear part of the distribution shown in Fig. 4a is used to evaluate the linear fit $\left(r^{2}>0.985\right)$ shown in Fig. 4 b. It can be evidenced that the linear trend is valid up to approximately $100 \mathrm{mmHg}$, which corresponds to an $A / A_{0}=1.1$ or $D / D_{0}=1.05$. At higher values of the pressure $p$, a deviation from the linear trend is detected. Stevanov et al. (2000) found a value of $80 \mathrm{mmHg}$ at a wall thickness of $1.04 \mathrm{~mm}$, up to which the dilatation was linear. These authors also realized the critical pressure level to increase with wall thickness, such that the present finding is consistent with their conclusion. The compliance determined experimentally in the present investigation is $27 \%$ lower than that predicted by Eq. 1. A similar tendency was observed by Stevanov et al. (2000) who showed the measured vessel compliance at $1.04 \mathrm{~mm}$ wall thickness was $12.3 \%$ lower than the predicted value.

The results in Fig. 4a further evidence the PDMS material to have a negligible hysteresis, i.e., the difference in the compliance between the load and unload cycles is within the measurement error. This is in good agreement with the findings in O'Sullivan et al. (2003). However, these authors evidenced a strong degradation as a material-aging effect. This result cannot be confirmed in the present compliance measurement, which is performed 28 days after curing. 
Fig. 4 a Cross-section area versus pressure for an elastic vessel with $h=1.55 \mathrm{~mm}$ and 28 days after curing. Values for increasing (plus sign) and decreasing (black dots) load cycles evidence negligible hysteresis. b Linear regime of (a)

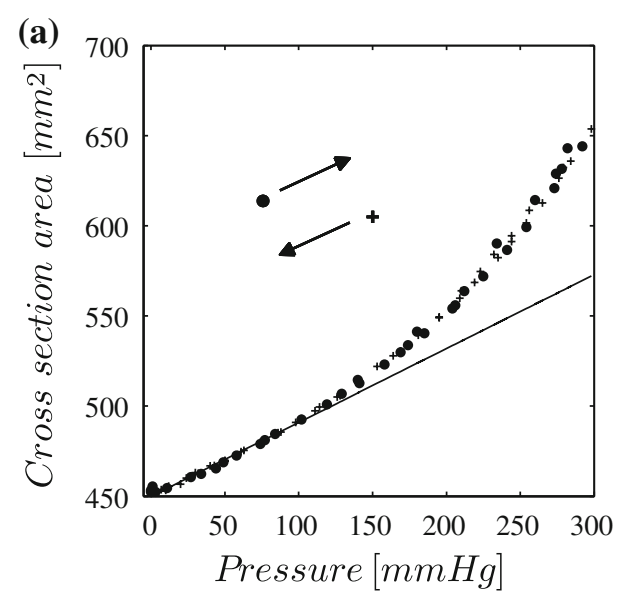

\section{The experimental setup}

\subsection{The flow facility}

A schematic of the flow facility is given in Fig. 5a. The flow facility is driven by a linear actuator (Moog D875), which can perform strokes up to $120 \mathrm{~mm}$ at frequencies up to $3 \mathrm{~Hz}$, such that the desired Reynolds and Womersley numbers can be realized. To achieve the necessary volumetric flow rates, a piston pump with an area of $31,400 \mathrm{~mm}^{2}$ is used such that volume of up to $3.81 \mathrm{can}$ be moved (Fig. 5b). The piston pump is connected to a $4.50-$ $\mathrm{m}$-long entrance length (Fig. 5c). The piston stroke and the cycle frequency can be independently adjusted such that several combinations of Reynolds numbers and Womersley numbers can be investigated. Note that, in this measurement campaign Reynolds numbers of $R e_{D}=1,000$ and 1,750 and Womersley numbers of 5 to 17.5 are analyzed.

This pipe has a $24 \mathrm{~mm}$ inner diameter, such that the entrance length corresponds to approximately $180 \mathrm{D}$. Many findings from the literature were compiled in Durst et al. (2005) and from additional experimental data the authors determined the necessary entrance length for fully developed steady laminar pipe flow at the Reynolds numbers in the present experiments to be $L / D=55-100$. Note that, this formula is derived for steady laminar flow and numerical calculations of Ünsal (2008) evidence the necessary entrance length to reduce by almost $40 \%$ at oscillating flow. As such, the entrance length of the pipe flow facility can be considered sufficient for fully developed flow state.

To achieve a homogeneous transition from the rigid entrance pipe to the flexible tube section custom-made adapters are developed. Figure 5d shows details of such an adapter. The adapter possesses a wedge-like shape with an inner diameter of $24 \mathrm{~mm}$ and a wedge angle of $10^{\circ}$. The flexible tube is sheathed over the wedge angle. The small value of the wedge angle is chosen to improve the homogeneity of the transition. A second adapter is used to tighten the flexible vessel in its position. To further ensure the flexible vessel to possess a homogeneous contour in the proximity of the transition and to not bulge, the second adapter guides the flexible vessel for another $8.2 \mathrm{~mm}$.

Since oscillating pipe flow is investigated, the exit of the vessel is connected to another rigid pipe section of $180 \mathrm{D}$ via a smooth transition adapter. This pipe section is connected to a reservoir (R1) with a sufficiently large surface area to ensure a constant static pressure level.

The test section (Fig. 5e), in which the flexible pipe section is positioned for RI matching, is also slightly pressurized using an additional reservoir (R2) connected to the test section. By adjusting the fluid elevation in the two reservoirs R1 and R2, the ambient pressure on the vessel can be varied. Hence, depending on the surface levels in the two reservoirs, different levels of transmural pressure difference can be achieved. The oscillating flow studies described in the context of this paper have been performed at a slightly positive transmural pressure, that is, at no flow the pressure inside the vessel is approximately $0.13 \mathrm{bar}$ higher than the ambient pressure inside the RI matching box. The RI matching box is approximately $800 \times$ $300 \times 100 \mathrm{~mm}$ in size such that the flexible vessel section and the junctions to the rigid entrance pipes can be placed within it. The streamwise position of the adapters can be varied such that the pretension of the flexible vessel can precisely be adapted to within approximately $1 \%$.

\subsection{Refractive index matching}

To achieve an optical transparency of the elastic vessel, the RI of the measurement fluid and of the fluid surrounding the vessel need to be adapted to that of the vessel material PDMS, which has been determined prior to the studies to be $n=1.406$. Therefore, the elastic tube is placed in a transparent PMMA box, which is filled with a water/ glycerine mixture of 60.7 weight percent glycerine ensuring 
Fig. 5 a Schematic of the test facility. b Linear actuator and piston pump. c Entrance and exit pipes. d Schematic of the adapters for the transition from the rigid into the flexible pipe sections. e Measurement section with RI matching box and optical measurement setup
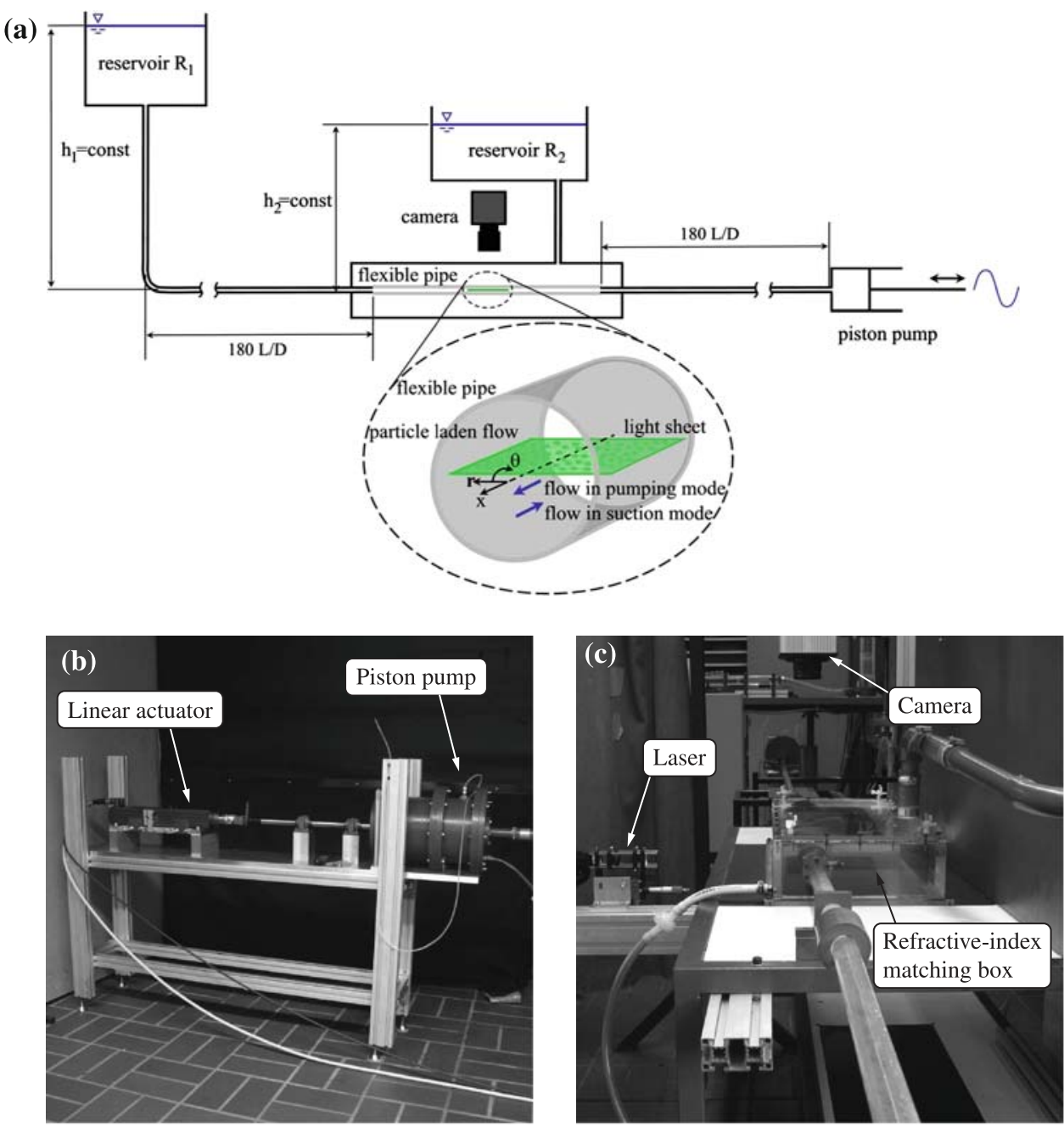

(d)

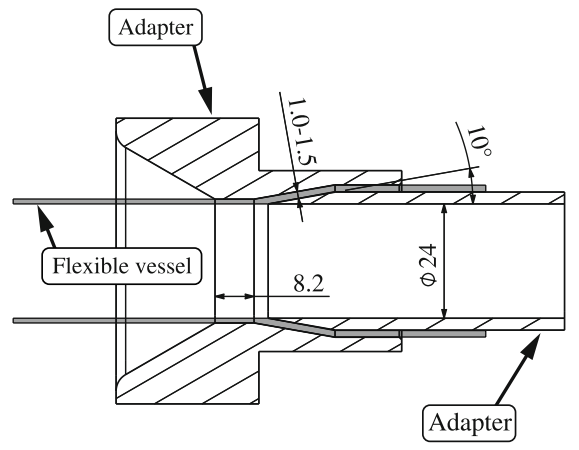

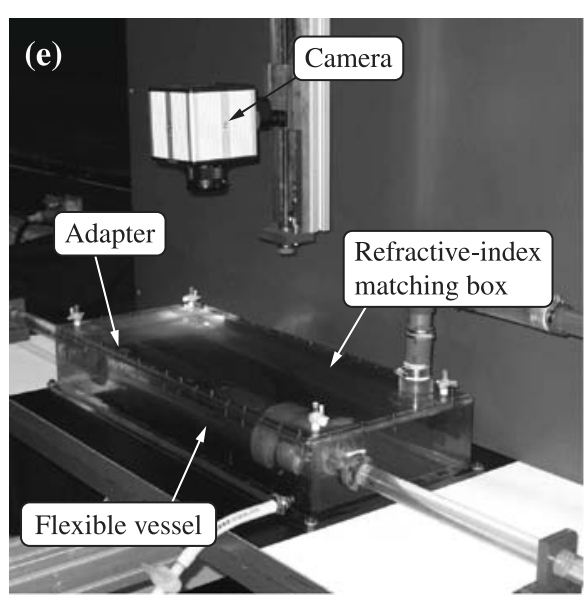

an optimum RI matching to the silicone-rubber. With the current setup, the fluid medium inside the vessel needs to be kept at 40.0 weight percent glycerine to fulfill the requirements regarding the physiological flow parameters. This was necessary since a plain water/glycerine solution was used during the first feasibility studies.
Dissolving e.g., sodium iodide (NaI) in a water/glycerine solution allows to adapt the RI and the density/viscosity simultaneously.

Especially in the case of the wall-shear stress determination from single pixel-line correlations, it is necessary that the optical access to the flow field in the vicinity of the 
vessel wall remains undistorted. Note that, while the streamwise direction and hence, the determination of the particle displacement stays unaffected by the optical distortion, the determination of the exact wall-normal position of the evaluation point can become strongly distorted. Since the wall-shear stress is determined from the nearwall velocity gradient, i.e., $\partial u /\left.\partial y\right|_{\text {wall }}$, also the wall-normal position needs to be assessed at high accuracy.

Let us in the following shortly review some aspects on the feasibility and sensibility of the RI matching. Narrow et al. (2000) report a temperature sensitivity of the RI of water enriched with sodium iodide of $\Delta n=0.0005 / \mathrm{K}$, which corresponds to $\approx 0.03 \% / \mathrm{K}$. The temperature sensitivity of the RI of pure water is $8.1 \times 10^{-5} / \mathrm{K}$ (Abbate et al. 1978). For water/glycerine the RI changes by $\Delta n=0.0015$ per percent increase in weight percent of glycerine in the mixture. Several authors report that RI matching can be performed as precise as $\Delta n=0.002$ to 0.005 (Miller et al. 2006; Narrow et al. 2000). Further details on possible RI matching and the experimental determination of the RI can be found in Budwig (1994), Miller et al. (2006), Narrow et al. (2000).

\subsection{PIV setup}

The flow field is investigated by high-speed PIV recordings with up to 1,000 samples at an effective frequency of $40 \mathrm{~Hz}$ at Reynolds numbers $\operatorname{Re}_{D}=1,000-1,750$ and Womersley numbers $\alpha=5-17.5$. Note that, in the case of the high Womersley number recordings, the effective frequency can be further increased for a higher temporal resolution. The PIV recordings can be synchronized with the piston stroke such that a proper phase-averaging of the results can be performed.

The light sheet plane is generated by a Darwin Duo high-speed laser and coincides with the centerline of the vessel. The plane can be adjusted with an angular misalignment in the order of $\pm 0.25^{\circ}$. The width of the light sheet is adjusted such that the complete field of view is homogeneously illuminated. The thickness of the laser sheet is minimized to approximately $0.5 \mathrm{~mm}$ and the constancy of the laser sheet thickness across the entire measurement section, i.e., perpendicular to the light sheet plane, is confirmed optically. The time between the laser pulses has to be adjusted in compliance with the flow field such that an optimum pixel displacement of the particles is achieved. Note that, at oscillating flow a compromise with respect to the remaining detection accuracy during flow reversal and with respect to the peak pixel displacements occurring at the cycle peaks is necessary.

The recording camera is a Photron Fastcam 1024 PCI camera with a resolution reduced to $1,024 \times 256 \mathrm{px}$ to allow for a higher total number of recorded images.
A 50-mm Nikkor AF with an f-number of $f^{\#}=1.8$ is used to reduce the depth of focus to a minimum and to maximize the available light. At the chosen magnification, the detection area is approximately $1.35 \times 0.35 D$, yielding a high enough optical resolution of the vessel. To be more precise, the undilated vessel diameter corresponds to approximately $750 \mathrm{px}$ on the recordings, such that the flow field can be derived with a resolution of up to $0.0013 \mathrm{D}$ applying singleline correlations, which will further be discussed in Sect. 5.2. It will be shown in Sect. 6 that this resolution can be considered high enough to accurately compute the near-wall velocity gradient to determine the wall-shear stress.

Polyamid (PA) particles with a mean diameter of $11 \mu \mathrm{m}$ are used as seeding material. The seeding is added prior to the measurements. To achieve a highly homogeneous distribution of the tracers, they are continuously added by a syringe while the flow facility is running.

The ability of the tracer particles of diameter $d_{p}$ and density $\rho_{p}$ to follow the fluid motion is characterized by the particle response time $\tau_{p}$. The particle response time can be calculated by the Basset-Boussinesq-Oseen equation (BBO) for the particle's behavior under acceleration (Melling 1997; Raffel et al. 2007). For PA particles $\rho_{p}=1.06 \mathrm{~g} / \mathrm{cm}^{3}$ such that $\tau_{p}=7 \mu \mathrm{s}$, which is considerably smaller than the typical time scales of the mean flow since they are in the order of the oscillation frequency. To determine the global flow field, the data are evaluated using commercial VidPIV ${ }^{\circledR} 4.6$ PIV software. The cross-correlation is based on standard FFT-based algorithms to calculate the particle displacement. A multi-pass correlation is applied starting with a $64 \times 64$ px interrogation size with $50 \%$ overlap followed by several adaptive cross-correlations with quadratic image deformation and B-Spline sub-pixel shifting with a final $24 \times 24$ px interrogation size with $50 \%$ overlap leading to a vector spacing of $0.016 \mathrm{D}$ and a flow field integration of $0.032 \mathrm{D}$. As mentioned earlier in this section, this resolution is insufficient to determine the wall-shear stress from the near-wall velocity gradient, such that the resolution needs to be further enhanced. Table 1 summarizes some of the measurement parameters.

Table 1 Overview of recording parameters

\begin{tabular}{ll}
\hline Field of view $(D)$ & $1.35 \times 0.35$ \\
Spatial resolution $(\mathrm{mm})$ & 1.28 \\
Spatial resolution $(D)$ & 0.032 \\
Vector spacing $(\mathrm{mm})$ & 0.64 \\
Vector spacing $(D)$ & 0.016 \\
Dynamic range $(\mathrm{px})$ & \pm 10.0 \\
Vectors/sample (-) & 1,350 \\
No. of samples (-) & $500-1,000$ \\
No. of cycles $(-)$ & $35-175$ \\
\hline
\end{tabular}




\section{Data evaluation}

Besides determining the flow field inside the vessel using PIV algorithms the vessel dilatation and the wall-shear stress can be computed from the measurements. In the following, the techniques applied to determine these latter variables will be discussed in detail. Furthermore, it is planned to determine the pressure gradient during the measurements. Here, this aspect will only shortly be addressed.

\subsection{Vessel dilatation}

Figure 6a exemplarily shows instantaneous recordings of the elastic vessel at three different times during the flow cycle, i.e., at three different vessel dilatations. The inner and outer contours of the vessel are clearly visible due to diffusive light reflections from the laser sheet. Using a magnification of the optical setup and depending on the combination of Reynolds and Womersley number vessel dilatations range from 5 to $30 \mathrm{px}$. The intensity distribution

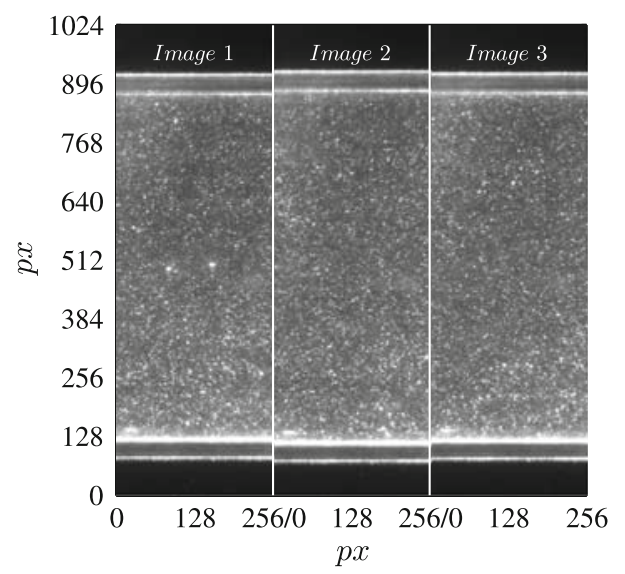

Fig. 6 Instantaneous PIV recordings at different dilatations of the elastic vessel

Fig. 7 a Exemplary gray-scale distribution along the contours of the vessels evidencing the vessel contours to be clearly distinguishable by their peaklike shape. b Remaining error in the determination of the vessel contour position of the vessel contour reflection is fitted to a Gaussian function, which is assumed to be representative for the position of the vessel-fluid interface.

To test the accuracy of the detection routines, representative images with a non-flexible, i.e., a rigid vessel have been recorded. Evaluating the position of the contour from several images allows to determine the precision, at which the contour can be detected, since the position of the structure should in the case of the rigid pipe be identical for all recordings.

Figure 7a shows an exemplary gray-scale distribution along the contours of the vessels, which shows the vessel contours to be clearly distinguishable by their peak-like shape. Figure $7 b$ evidences to what degree the position of the vessel contour can be determined by the above-mentioned scheme. A sequence of 50 images has been evaluated and the results at different vessel contour positions in the image evidence the routines to allow the determination of the vessel contour position with a remaining scatter in the order of approximately $\pm 0.5 \mathrm{px}$, with an rms of the deviation of $0.13-0.15$. Note that, the mean pixel position of the vessel contour has been subtracted in Fig. 7b, such that the deviation from zero indicates the precision, at which the vessel contour is determined. By averaging the positions of up to 20 neighboring pixel lines along the vessel axis, the rms value of the deviation in determining the contour can be reduced to approximately $0.05-0.07 \mathrm{px}$. At the chosen magnification, 20 px correspond to approximately $0.026 \mathrm{D}$ such that the vessel dilatation along such distances can be assumed constant. As stated earlier, the maximum vessel dilatations during the flow cycle range from approximately 5-30 px such that the error in determining the vessel dilatation is in the order of $0.5-3.0 \%$.

Since the inner and outer vessel contours can be detected, changes in the local wall thickness, which are due to the dilatation of the vessel can also be determined.

Figure 8a shows exemplary gray-scale distributions similar to that in Fig. $7 \mathrm{a}$ at different times during the
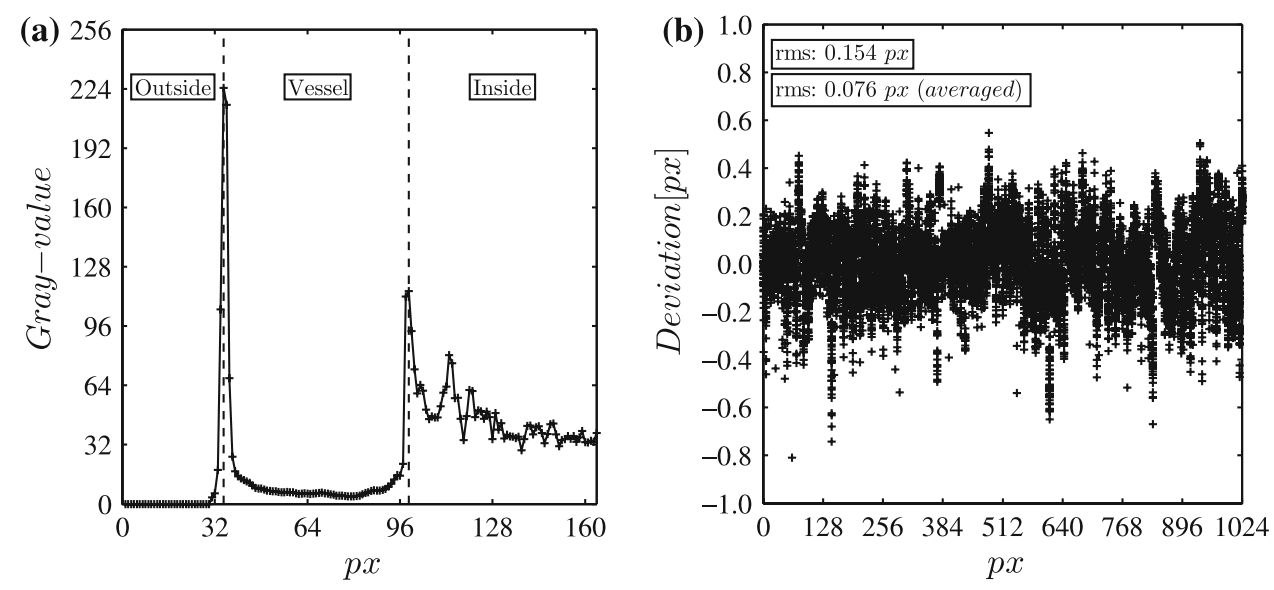
Fig. 8 a Exemplary gray-scale distributions for several time steps at oscillating flow in an elastic vessel. b Collapse of the shifted gray-scale distributions

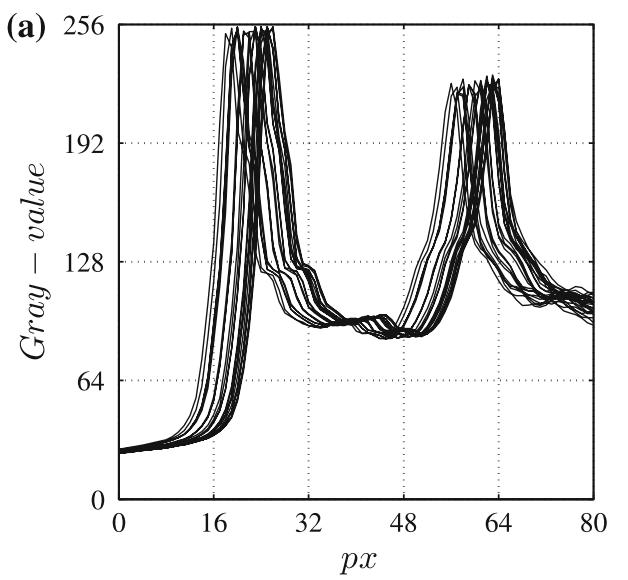

oscillating flow cycle for an elastic vessel. It is evident that the position of the peaks varies by up to $8 \mathrm{px}$ at the chosen experimental conditions. Figure $8 \mathrm{~b}$ shows the same distributions, shifted along the lateral direction by the value of the displacement determined for the outer vessel contour position (left peak). The distributions collapse quite well on a single line, which also indicates the gray-scale distribution of the scattered light to not strongly change during oscillation. A slight scatter of the distributions at the inner vessel contour (right peak) is evident. This is rather related to the contributions of the near-wall particles in the flow field than being caused by a change in the thickness of the material during the oscillation cycle.

\subsection{Wall-shear stress}

The wall-shear stress results from the relative motion between the fluid and the vessel wall. The determination of wall-shear stress is of utter importance in many biological flows and especially in vascular research (Hierck et al. 2008) and it is expected that the flexibility of the vessel wall strongly effects the wall friction (Poelma et al. 2008). The wall-shear stress is defined as

$\tau_{\text {wall }}=\left.\eta \frac{\partial u}{\partial y}\right|_{\text {wall }}$,

where $\eta$ is the dynamic viscosity of the flow and $\partial u /\left.\partial y\right|_{\text {wall }}$ the velocity gradient at the wall. That is, to determine the instantaneous wall-shear stress from the flow-field measurements it is necessary to assess the velocity gradient in the extreme vicinity of the wall. In oscillating flows this is difficult since the flow field in the near-wall region is strongly curved (Womersley 1955, 1957a, b) and consequently, the evaluation of the wall-shear stress requires velocity data very close to the wall, where the gradient can still be considered constant.

Analytical investigations further explained in Sect. 6 evidence that the velocity gradient is to be detected within
$0.005 D$ to the wall to obtain a velocity gradient distribution, which can be considered representative for the wallshear stress in phase and amplitude. That is, the optical resolution obtained by PIV applying interrogation windows of e.g., 24 px is not sufficient, since the integration of a non-linear velocity gradient in the interrogation window would strongly deteriorate the estimate of the near-wall velocity gradient. Note that, due to the phase dependence of the flow field in the proximity of the wall, the integration of the flow field within a standard PIV interrogation window causes strong deviations (Westerweel 2008) of the wall-shear stress determined from the velocity gradient.

Therefore, instead of using two-dimensional interrogation windows as in PIV, the velocity gradient in the vicinity of the wall is computed from particle displacements along single, wall-parallel pixel lines in the recorded images. In doing so, the velocity profile is determined down to approximately $0.0013 \mathrm{D}$, which is less than the abovementioned necessary limit.

The correlation is performed by multiplying the Fast Fourier transforms (FFT) of the one-dimensional grayscale distributions of a single pixel line. Consequently, a Gaussian fit of type $a \cdot \exp \left(-((x-b) / c)^{2}\right)$ is applied to the inverse FFT, i.e., the correlation of the signals, to obtain the shift of the wall-parallel gray-scale distribution between two consecutive images. The procedure thereby follows that of modern PIV algorithms, except that it is applied to one-dimensional data instead of two-dimensional gray-scale distributions as in the case of standard interrogation windows.

Figure 9a exemplarily shows two such consecutive gray-scale distributions along a single pixel line with the corresponding correlation and the Gaussian fit of the correlation maximum. Typically, the length of the pixel line is set to $128-256 \mathrm{px}$ to attain a reliable estimate of the shift. In the case of non-uniform displacements of the vessel wall (e.g., measurements of stenosis flow), this length needs to be reduced to regions, in which a constant displacement 
Fig. 9 a Exemplary near-wall gray-scale distributions with the corresponding correlation and the Gaussian fit. b Tests of the correlation routines using generic PIV data. The deviation of the detected single-line grayscale displacement from the accurate shift in the generic data and the rms of the error to determine the deviation normalized by the value of the generic displacement $\mathrm{rms} / \mathrm{disp}$. are given. Results are based on 64 independent realizations (a)
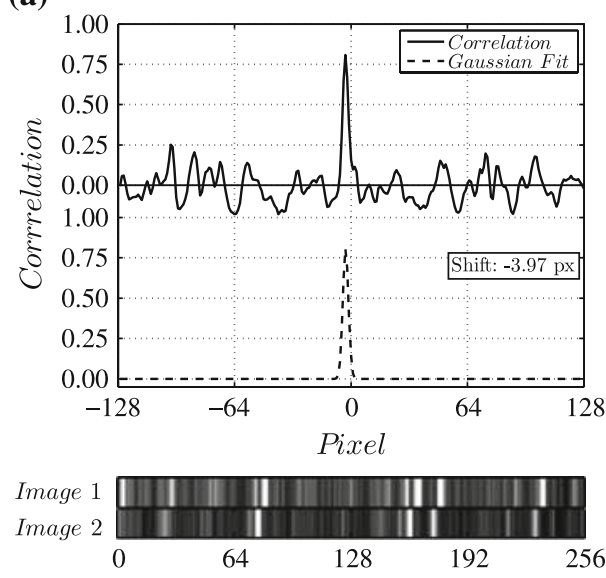

(b)

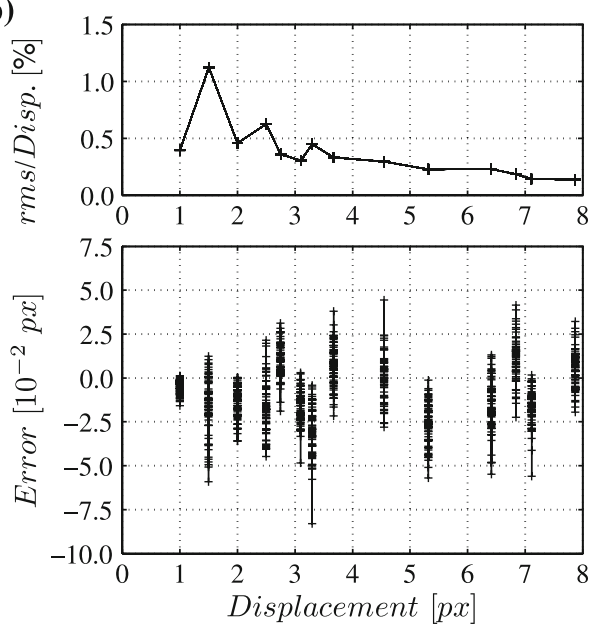

can still be assumed. At the current optical magnifications, a length of $128-256$ px only corresponds to $0.15-0.3 \mathrm{D}$. To apply the above procedure to non-uniform dilations of the vessel, it might become necessary to rotate the image such that locally pixel lines follow the vessel contour. This procedure is explained in great detail e.g., in (Theunissen et al. 2008).

The accuracy of the algorithms is tested by evaluating artificial particle fields. The seeding quality and particle density of the generic images is chosen similar to those obtained during the measurements. Several images with different sub-pixel particle shifts ranging from 0.00 to $8.00 \mathrm{px}$ are generated. A representative result obtained from the evaluation of 64 independent images is given in Fig. 9b. It evidences the deviations of the detected single-line grayscale displacements from the actual one to be approximately $\pm 0.05 \mathrm{px}$. The rms of the error normalized by the generic displacement of the deviation rms/disp. is below $1.5 \%$ at all investigated generic displacements. Typical displacements in the near-wall field range between $\pm 1.5 \mathrm{px}$, the differential increase in the pixel displacement between consecutive pixel lines along the wall-normal direction in the vicinity of the wall, i.e., within the first pixel lines, has been determined to be approximately $0.5 \mathrm{px}$ at the present fluid-mechanical settings and the chosen optical setup. This difference in pixel displacement between consecutive pixel lines is nearly constant, indicating a rather linear velocity gradient in this region. Currently, this allows to determine the velocity field and as such the wall-shear stress at an overall accuracy of less than 5\%.

Applying multi-passing correlation routines and fitting the correlation peak with a Whittaker function might further increase the accuracy of the routines. However, currently the achievable accuracy is considered sufficient.

Instead of deriving the wall-shear stress directly at the wall, it is useful to obtain the wall-shear stress from the velocity gradient in the vicinity of the wall. That is, the wall-shear stress is determined by the difference between two adjacent pixel lines, e.g., the first and second pixel lines, which are completely positioned within the flow field. These lines can be identified from the coordinates of the vessel contour determined via the technique described above. By using this technique the ambiguity of finding the exact position of the vessel contour, i.e., the position, at which the velocity becomes zero, can be avoided. It only has to be made sure that the wall-normal distance between single pixel lines in the near-wall region is not optically distorted due to slight changes in the RIs. This will further be discussed at the end of Sect. 6.

\subsection{Pressure drop}

Pressure taps in the proximity of the adapters on both sides of the flexible vessel will allow to simultaneously determine the pressure drop during the optical flow measurements. Up- and down-stream of the flexible vessel section $0.5 \mathrm{~mm}$ pressure taps are positioned in the rigid pipes. Kulite XCL-100 pressure transducers with a diameter of $2.6 \mathrm{~mm}$ and a nominal pressure range of $0-1$ bar are used for the measurements. The pressure transducers possess a linearity of $\pm 0.1 \%$ FSO and hysteresis and repeatability of $\pm 0.5 \%$ FSO. They are connected to A/D-devices (AW980, Schyschka-Elektrik).

Furthermore, the local pressure at the measurement position will be investigated. A close-up of the modification of the vessel necessary for the local pressure measurements is given in Fig. 10a and an image of a vessel with an appropriate adapter is given in Fig. 10b. Note, however, that further tests are necessary to prove the existence of the adapter to not influence the symmetry of the flow field and of the vessel dilatation. 
Fig. 10 a Schematic of the planned adapter used for pressure measurements in the elastic vessel. b Vessel with adapter and pressure transducer (a)

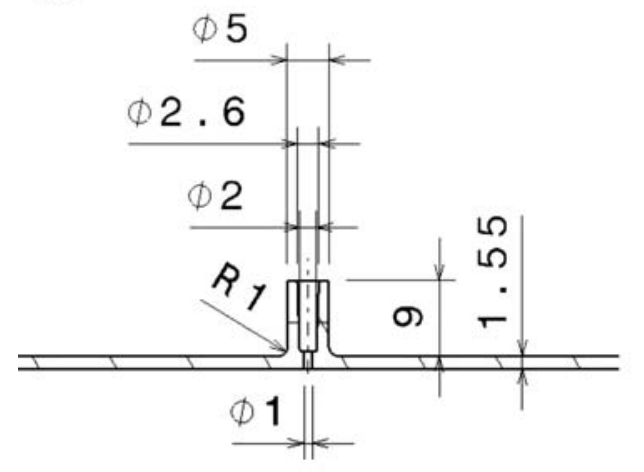

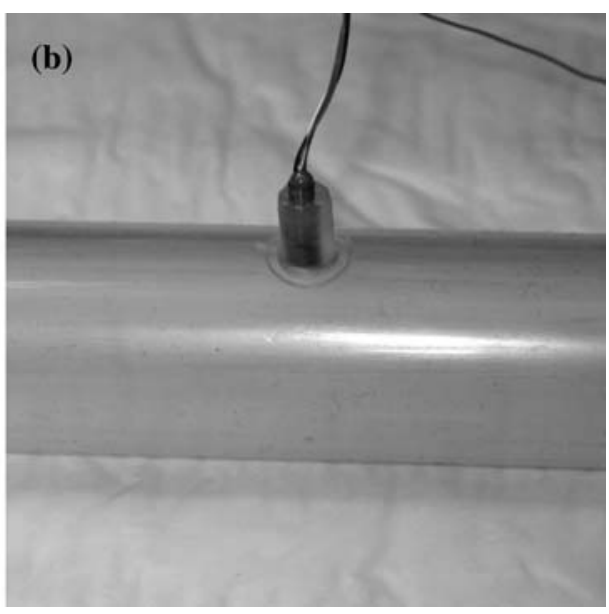

\section{Validation of the measurement technique/flow field in rigid tubes}

To confirm the flow conditions of the oscillatory laminar flow inside the vessel and to further evaluate the ability to determine the flow field by the techniques described in Sect. 5, measurements of the flow field in a rigid Plexiglas tube under identical conditions to those of the oscillating flow in the flexible vessel are performed. Note that, these measurements have not been performed with the aforementioned high-speed PIV system but applying a standard PIV equipment at recordings frequencies of $2-5 \mathrm{~Hz}$. Since the analytical solution for laminar oscillating pipe flow is well known, the experimental results can be compared to the analytical Womersley solution.

Exemplary results of the mean velocity distribution at $R e_{D}=1,000$ and $\alpha=12.5$ are presented in Fig. 11a at several phase angles with an increment of $45^{\circ}$. The experimentally obtained distributions are compared with the analytical solution and evidence good agreement at all phase angles. Furthermore, Fig. 11b evidences a high degree of symmetry of the velocity profiles. The velocity profiles of the left and right half perfectly collapse.
Likewise good agreement can be found at other combinations of Womersley and Reynolds number. To show the quality, at which the wall-shear stress can be determined from the near-wall velocity gradient, again experimental data are compared with the analytical Womersley solution. In Fig. 12a exemplary results of temporal distributions of the near-wall velocity gradient, i.e., a direct representative of the wall-shear stress $\tau_{\text {wall }}$, and of the centerline velocity at $R e_{D}=1,000$ and $\alpha=5$ are compared with analytical distributions. A phase shift between the distributions evidences the near-wall velocity to precede the fluid motion at the centerline.

Figure $12 b-d$ shows the tendency of the phase shifts at several Reynolds numbers as a function of the wall distance which is used to determine the wall-shear stress. The theoretical asymptotic value given in the figures is derived from the analytical Womersley solution with a spatial discretization of $0.0017 \mathrm{D}$, which can be shown to be high enough to achieve a representative value for the wall-shear stress with respect to amplitude and phase lag. The errorbars shown in the figure represent the rms value of phase angle determined from approximately five independent temporal sequences. It is evident that the velocity needs to
Fig. 11 a Mean velocity profiles compared with the analytical Womersley solution for laminar oscillating pipe flow at phase angles shifted by $45^{\circ}$. b Velocity distributions at different phase angles. Note that the mirror image of the left half of the velocity distribution is projected onto the right half velocity distribution
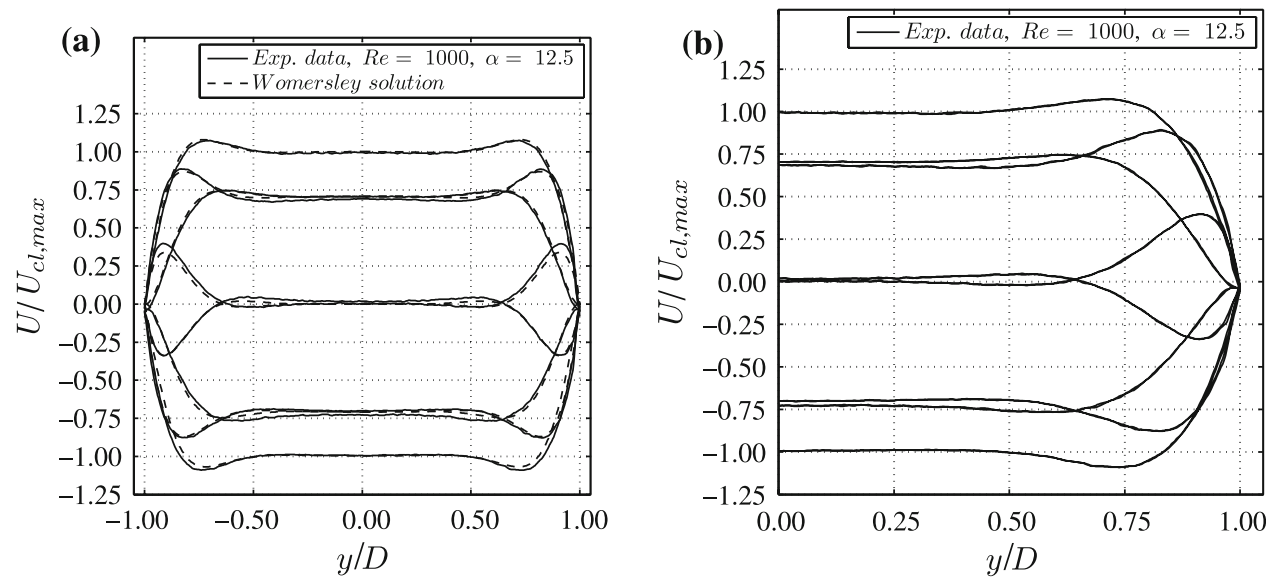
Fig. 12 a Exemplary results of the centerline velocity and the near-wall velocity for laminar oscillating pipe flow at $\operatorname{Re}_{D}=1,000$ and $\alpha=5$. b, $\mathbf{c}, \mathbf{d}$ Phase shift $\Phi_{\tau_{\text {wall }}}-\Phi_{U_{c l}}$ as a function of wall distance, at which the wall-shear stress is determined at different combinations of Womersley and Reynolds numbers
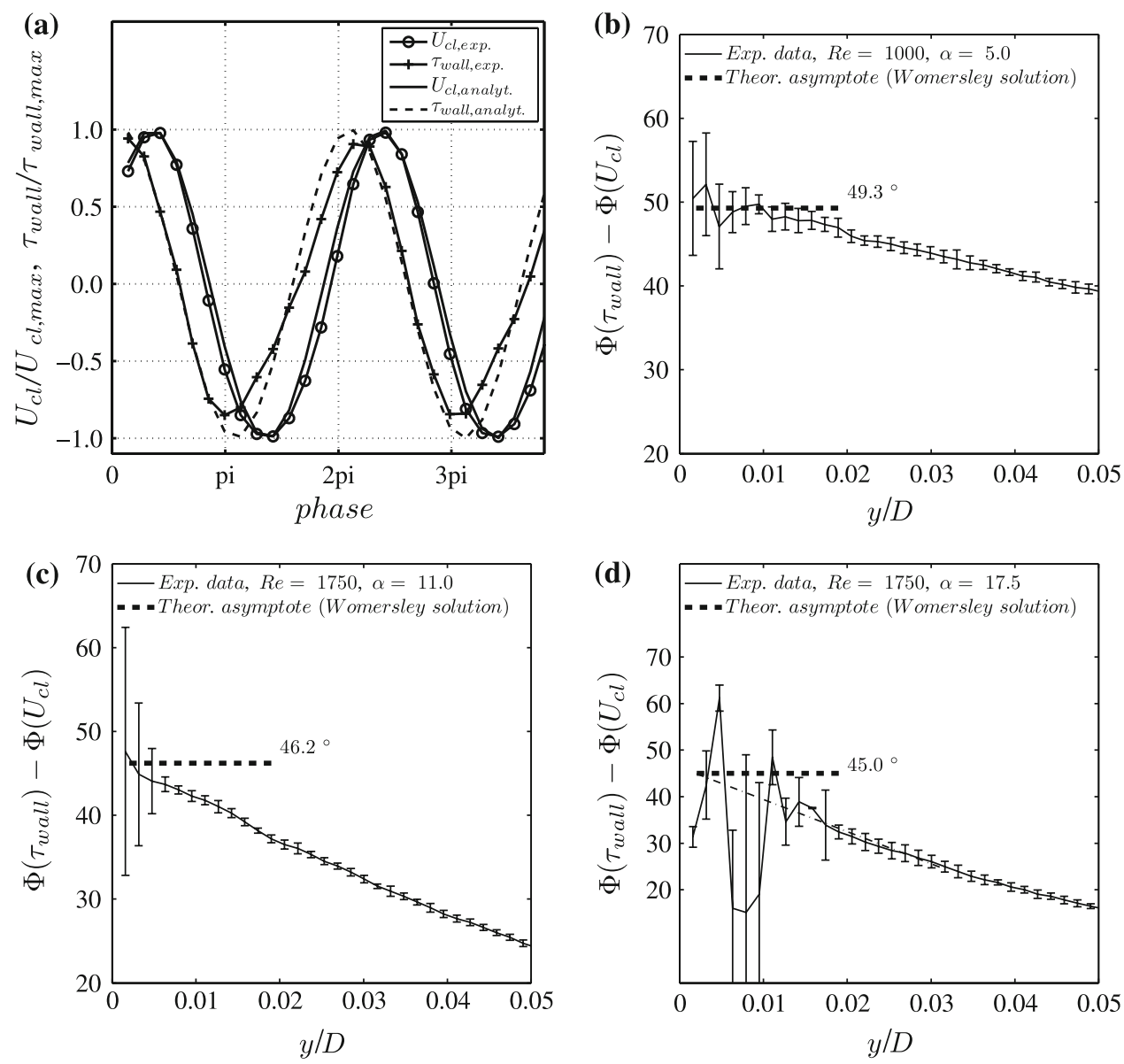

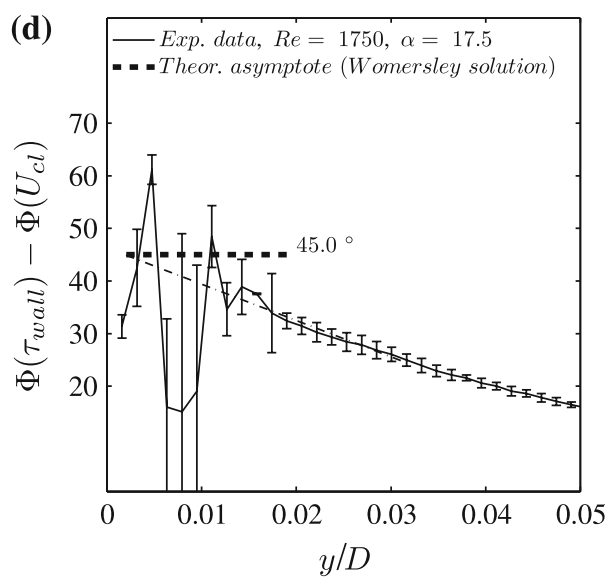

be determined in the extreme proximity of the wall to obtain a reliable estimate of the wall-shear stress. To be more precise, an adequate determination is only possible within the first 1-3 px from the wall. At higher wall distances, the phase lag slowly starts to monotonically decrease.

Note, the high level of scatter in the proximity of the wall evident in Fig. 12d results from a poor quality of the PIV images in the proximity of the wall. This was mainly caused by a high amount of particles stuck to the wall. Furthermore, it needs to be kept in mind that, the RI matching in the case of the rigid pipe is inferior to that with the PDMS flexible vessel since the water/glycerine mixture much better adapts to the RI of PDMS than to that of PMMA causing higher levels of reflections in the case of the rigid pipe. However, the results at lower Womersley numbers and the linear trend, which is fitted to the data in Fig. 12d indicate the applied technique to achieve a sufficient resolution of the near-wall velocity field necessary to determine the wall-shear stress.

In the case of an ideal RI-matching of the working fluid with the vessel material and the fluid in the RI-matching box, the accuracy in determining the phase lag and the amplitude of the wall-shear stress from the near-wall velocity gradient increases with the optical discretization of the fluid region, i.e., with optical magnification. In Fig. 13a, the error in the phase lag of the wall-shear stress determined from the near-wall velocity gradient at finite discretization and that derived from the analytical Womersley solution can be estimated to be approximately $0.2^{\circ}-0.7^{\circ}$ at Womersley numbers of $\alpha=5-17.5$ (Fig. 13a). The error in estimating the amplitude of the wall-shear stress from the near-wall velocity gradient (Fig. 13b) becomes

$\frac{\left.(\partial u / \partial y)\right|_{\text {analyt. }}-(\partial u / \partial y)}{\left.(\partial u / \partial y)\right|_{\text {analyt. }}} \leq 0.4 \%$.

It is $0.3-1.0 \%$ at the Womersley numbers in the current experiments. The results indicate that at the Womersley numbers ranging from $\alpha=5-17.5$, the velocity gradient can be performed sufficiently close to the wall such that the near-wall velocity gradient can be considered representative of the wall-shear stress.

Realistically, the RI-matching can only be performed within approximately $\Delta n=0.005$ (see Sect. 4.2). To investigate the influence of optical aberrations in the nearwall region due to insufficient RI-matching, the following considerations should help. As mentioned earlier, the 


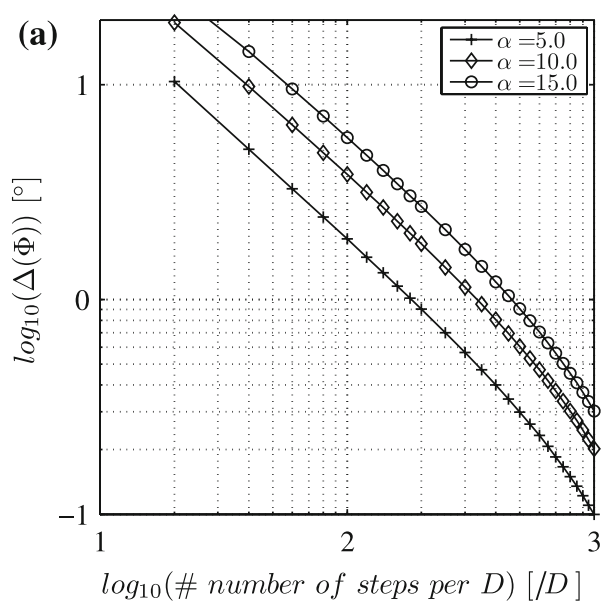

Fig. 13 a Remaining phase error in degrees in determining the wallshear stress from the near-wall velocity gradient as a function of the spatial discretization of the analytical Womersley solution. b Normalized error $\left.\left(\left.(\partial u / \partial y)\right|_{\text {asympt. }}-\partial u / \partial y\right)\right) /\left.(\partial u / \partial y)\right|_{\text {asympt. }}$ for

wall-shear stress $\tau_{\text {wall }}=\eta \partial u / \partial y$ is determined from the near-wall velocity gradient. Furthermore, the estimate of the wall-shear stress is not determined directly at the wall, but rather from the velocity gradient in a region $0.005 \mathrm{D}$ from the wall. The wall-parallel velocities are not affected by optical aberrations due to the curvature of the vessel. On the other hand, dy, over which the velocity gradient is determined, is influenced by the mismatch of the RIs. To be more precise, the near-wall velocity field is stretched along the wall-normal direction. In Fig. 14, the resulting wallnormal stretching/magnification due to realistic discrepancies in the RIs of the working fluid and the vessel material is given. The discretization of the velocity field in the vessel has been chosen similar to that in the experiments. It is evident from Fig. 14a, that almost through the entire flow field the magnification is less than 1.025. Only in the extreme proximity of the wall-approximately the first 2 pixel at the current optical setup-the magnification

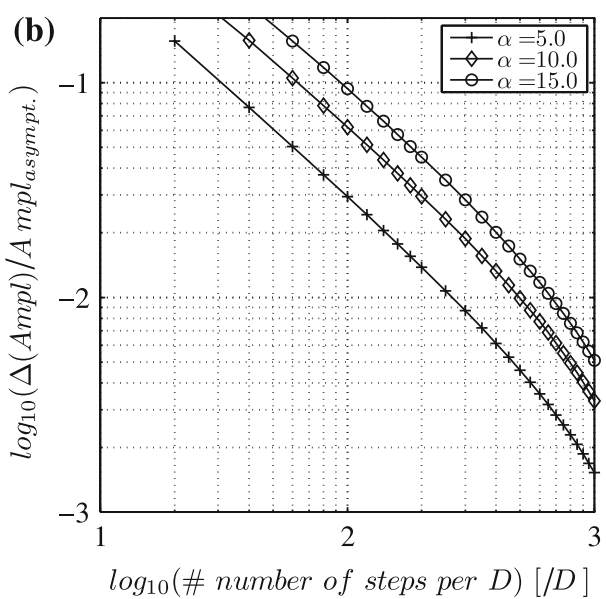

determining the amplitude of the wall-shear stress from the nearwall velocity gradient as a function of the spatial discretization of the analytical Womersley solution

increases to $1.05-1.15 \%$. The local increase in magnification, i.e., its linearity, is given in Fig. 14b. Again, it is evident, that the magnification is nearly constant (linearity value approximately 0 ) for $r / R_{i} \leq 0.99$. Only in the vicinity of the wall, the magnification increases strongly. That is, as long as the wall-shear stress is determined at wall distances $r / R_{i} \approx 0.99 \equiv r / D=0.995$, the error due to the local magnification is within $2.5 \%$. This wall distance was earlier determined to be still adequate to also determine the phase of the wall-shear stress correctly.

\section{Conclusion}

The paper presents a technique to manufacture elastic vessels with tunable geometrical and mechanical properties resembling physiological relevant vessels. Furthermore, an optical test procedure is explained to precisely measure the
Fig. 14 a Optical magnification (due to wall-normal stretch) of the flow field in the vicinity of the wall. b Non-linearity of the magnification (due to wallnormal stretch) of the flow field in the vicinity of the wall
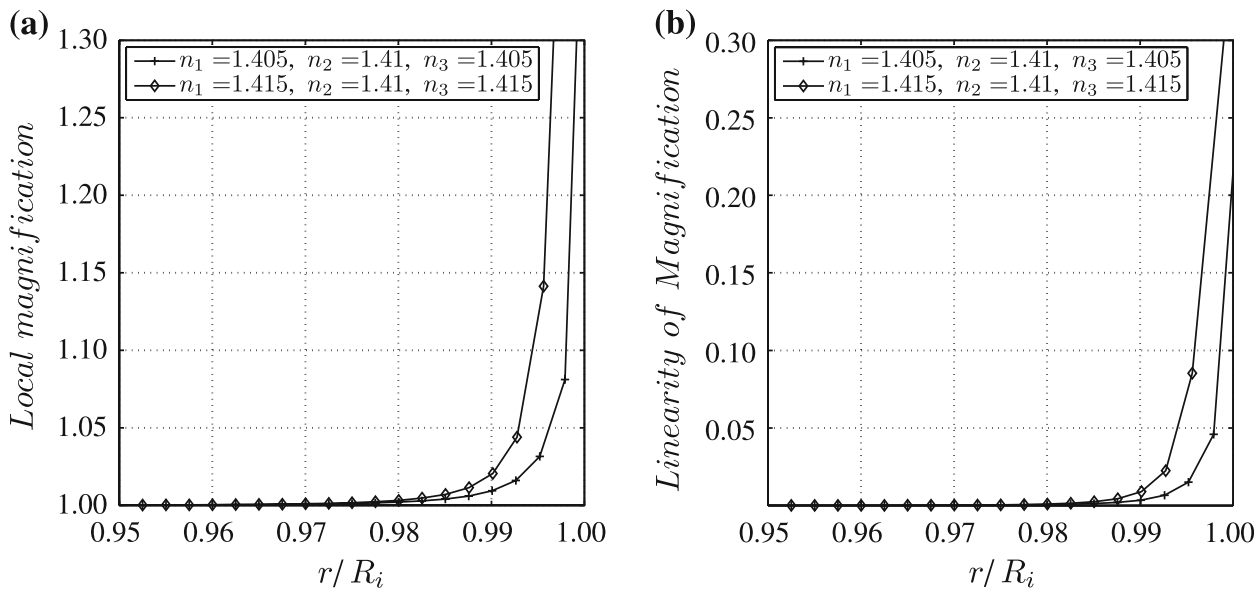
flow field, the dilatation of the vessel walls, and the wallshear stress of oscillating flow in an elastic vessel, the latter variable being of utter importance in vascular research.

Several techniques have been presented allowing to validate the characteristics of the elastic vessel, which is of high importance since these characteristics serve as boundary conditions of the behavior of the elastic vessels. In the future it is planned to investigate how far the anisotropic character of real vascular vessels can be mimicked.

It has been shown that the RI-matched facility in combination with the PIV technique allows to precisely detect the wall of the vessel, i.e., the vessel dilatation, simultaneously with the measurement of the flow field inside the vessel. In general, the measurements of the flow field inside the vessel show good agreement with the analytical solution in terms of symmetry, velocity distribution, phase angles, and velocity gradients.

Furthermore, the ability to determine the wall-shear stress by calculating the velocity gradient at the wall using a highly resolved velocity distribution close to the wall, which is obtained by a single-pixel line correlation, has been shown. The single-pixel line correlation technique has been validated by applying it to data of oscillating flow inside a rigid pipe and by comparing the results to the analytical Womersley solution. The comparison indicates the applied technique to yield a reliable estimate of the wall-shear stress. In future studies we will investigate in how far a polynomial fit of the near-wall velocity gradient will help to further increase the reliability of the technique.

Further investigations using the validated measurement techniques of oscillating flow in an elastic vessel at Womersley numbers of 5-17.5 and Reynolds numbers of $1,000-1,750$ will be presented in a subsequent publication. The ability of the presented measurement technique to provide accurate results for vessel dilatation, velocity fields and wall-shear stress distributions has been proven and discussed in detail in the present paper.

Acknowledgments The authors would like to thank Dr. G. A. Krombach from the Department for Radiological Diagnostics of the University Hospital Aachen for imaging the vessels using Magnetic Resonance Tomography (MRT).

Open Access This article is distributed under the terms of the Creative Commons Attribution Noncommercial License which permits any noncommercial use, distribution, and reproduction in any medium, provided the original author(s) and source are credited.

\section{References}

Abbate G, Bernini U, Ragozzino E, Somma F (1978) The temperature dependence of the refractive index of water. J Phys D Appl Phys 11:1167-1172
Armani D, Liu C (1998) Re-configurable fluid circuits by PDMS elastomer micromachining. In: Proceedings of the 12th international conference on MEMS 99, Orlando, Florida, USA, pp 222227

Budwig R (1994) Refractive index matching methods for liquid flow investigations. Exp Fluids 17:350-355

Dow Corning Corporation (1991) Information about high technology silicone materials. Technical report, Dow Corning Corporation

Durst F, Ray S, Ünsal B, Bayoumi OA (2005) The development lengths of laminar pipe and channel flows. J Fluids Eng Trans ASME 127:1154-1160

Eguchi T, Watanabe S, Takahara H, Furukawa A (2003) Development of pulsatile flow experiment system and PIV measurement in an elastic tube. Memoirs of the Faculty of Engineering, Kyushu University 63:161-172

Große S, Schröder W, Klaas M, Klöckner A, Roggenkamp J (2007) Time resolved analysis of steady and oscillating flow in the upper human airways. Exp Fluids 42(6):955-970. doi:10.1007/s00348007-0318-y. http://dx.doi.org/10.1007/s00348-007-0318-y

Große S, Schröder W, Klaas M (2008) Time-resolved PIV measurements of vortical structures in the upper human airways. In: Schröder A, Willert C (eds) Topics of applied physics, vol. 112: Particle image velocimetry-new developments and recent applications. Springer, Berlin, pp 35-53. doi:10.1007/978-3540-73528-1. http://dx.doi.org/10.1007/978-3-540-73528-1

Hale JF, McDonald DA, Womersley JR (1955) Velocity profiles of oscillating arterial flow, with some calculations of viscous Drag and the Reynolds number. J Physiol 128:629-640

Hierck BP, Van der Heiden K, Poelma C, Westerweel J, Poelmann RE (2008) Fluid shear stress and inner curvature remodeling of the embryonic heart. Choosing the right lane! Sci World J Cardiac Dev 8:212-222

Lötters JC, Olthuis W, Veltink PH, Bergveld P (1996) Polydimethylsiloxane as an elastic material applied in a capacitive accelerometer. J Micromech Microeng 6:52-54

Lötters JC, Olthuis W, Veltink PH, Bergveld P (1997a) Polydimethylsiloxane, a photocurable rubberelastic polymer used as spring material in micromechanical sensors. Microsyst Technol $3(2): 64-67$

Lötters JC, Olthuis W, Veltink PH, Bergveld P (1997b) The mechanical properties of the rubber elastic polymer polydimethylsiloxane for sensor applications. J Micromech Microeng 7:145-147

McDonald DA (1955) The relation of pulsatile pressure to flow in arteries. J Physiol 127:533-552

Melling A (1997) Tracer particles and seeding for particle image velocimetry. Meas Sci Technol 8:1406-1416

Mijovic B, Liepsch D (2003) Experimental flow studies in an elastic Y-model. Technol Health Care 11:115-141

Miller P, Danielson K, Moody G, Slifka A, Drexler E, Hertzberg J (2006) Matching index of refraction using a diethyl phthalate/ ethanol solution for in vitro cardiovascular models. Exp Fluids 41:375-381

Narrow TL, Yoda M, Abdel-Khalik SI (2000) A simple model for the refractive index of sodium iodide aqueous solutions. Exp Fluids 28(3):282-283

O'Sullivan S, Nagle R, McEwen JA, Casey V (2003) Elastomer rubbers as deflection elements in pressure sensors: investigation of properties using a custom designed programmable elastomer test rig. J Phys D Appl Phys 36(15):1910-1916

Poelma C, Vennemann P, Lindken R, Westerweel J (2008) In vivo blood flow and wall shear stress measurements in the vitelline network. Exp Fluids 45(4):703-713

Raffel M, Willert CE, Wereley ST, Kompenhans J (2007) Particle image velocimetry-a practical guide. Springer, Berlin 
Stevanov M, Baruthio J, Eclancher B (2000) Fabrication of elastomer arterial models with specified compliance. J Appl Physiol 88:1291-1294

Theunissen R, Scarano F, Riethmuller ML (2008) On improvement of PIV image interrogation near stationary interfaces. Exp Fluids 45: $557-572$

Ünsal B (2008) Time-dependent lamiar, transitional and turbulent pipe flows. PhD thesis, Universität Erlangen-Nürnberg

van Hout JI, Scheurer J, Casey V (2003) Elastomer microspring arrays for biomedical sensors fabricated using micromachined silicon molds. J Micromech Microeng 13:885-891
Westerweel J (2008) On velocity gradients in PIV interrogation. Exp Fluids 44(5):831-842

Womersley JR (1955) Method for the calculation of velocity, rate of flow and viscous drag in arteries when the pressure gradient is known. J Physiol 127:553-563

Womersley JR (1957a) An elastic tube theory of pulse transmission and oscillatory flow in mammalian arteries. WADC Technical report TR 56-614

Womersley JR (1957b) Oscillatory flow in arteries: the constrained elastic tube as a model of arterial flow and pulse transmission. Phys Med Biol 2:178-187 